\title{
Current Procedural Terminology: History, Structure, and Relationship to Valuation for the Neuroradiologist
}

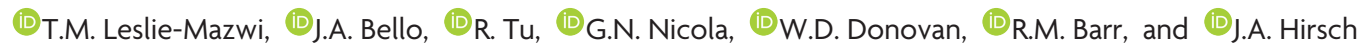

\begin{abstract}
SUMMARY: The year 1965 was critical for US health care policy. In that year, Medicare was created as part of the Social Security Act under President Lyndon B. Johnson after several earlier attempts by Presidents Franklin Roosevelt and Harry Truman. In 1966, the American Medical Association first published a set of standard terms and descriptors to document medical procedures, known as Current Procedural Terminology, or CPT. Fifty years later, though providers have certainly heard the term "CPT code," most would benefit from an enhanced understanding of the historical basis, current structure, and relationship to valuation of Current Procedural Terminology. This article will highlight this evolution, particularly as it relates to neuroradiology.
\end{abstract}

ABBREVIATIONS: AMA = American Medical Association; ASNR = American Society of Neuroradiology; CMS = Centers for Medicare and Medicaid Services; $\mathrm{CPT}=$ Current Procedural Terminology; RUC = AMA/Specialty Society Relative Value Scale Update Committee; RVU = relative value units

$\mathbf{P}$ resident Lyndon B. Johnson's “Great Society” programs included the creation of Medicare in 1965, which, among its many facets, highlighted the need for efficient and effective determination of physician reimbursement. Consistent and accurate reimbursement requires a common language of medical procedures. The American Medical Association (AMA) plays a unique and sometimes underappreciated physician-centric role in the determination of provider reimbursement. In 1965, the AMA recognized the need for common language and helped create the Current Procedural Terminology (CPT) system to describe medical services and procedures.

The first edition of CPT was published in 1966 and, for the most part, was focused on surgery. As a result, neuroradiology had little representative coding. At that time, 4-digit codes were used. The second edition, published in 1970, expanded each code to 5 digits, with the 70000-79999 code series representing radiology. In the mid- and late 1970s, the third and fourth editions of CPT were released. Not surprisingly, each version was progres-

Received April 2, 2016; accepted after revision May 9.

From the Neuroendovascular Program (T.M.L.-M., J.A.H.), Massachusetts General Hospital, Boston, Massachusetts; Montefiore Medical Center (J.A.B.), Albert Einstein College of Medicine, Bronx, New York; Progressive Radiology (R.T.), The George Washington University, Falls Church, Virginia; Hackensack University Medical Center (G.N.N.), Hackensack, New Jersey; Norwich Diagnostic Imaging Associates (W.D.D.), Norwich, Connecticut; and Mecklenburg Radiology Associates P.A. (R.M.B.), Charlotte, North Carolina.

Please address correspondence to Thabele M. Leslie-Mazwi, Neuroendovascular Program, Massachusetts General Hospital, Harvard Medical School, Boston, MA 02114; e-mail: tleslie-mazwi@mgh.harvard.edu; @JoshuaAHirsch

http://dx.doi.org/10.3174/ajnr.A4863 sively more detailed, in line with the increasingly complex health care system. This evolution has continued since. In 1983, the predecessor of the Centers for Medicare and Medicaid Services (CMS) merged CPT into the Healthcare Common Procedure Coding System, which historically had performed a similar function to CPT. Just as the International Classification of Diseases published by the World Health Organization maintains an updated classification of medical conditions, CPT maintains an updated classification of medical procedures. ${ }^{1-3}$

Thirty years after the creation of the CPT system, the KennedyKassebaum Act, better known as the Health Insurance Portability and Accountability Act of 1996, pushed the legacy of CPT forward. ${ }^{4}$ That legislation required the Department of Health and Human Services to set up standards for the electronic transaction of health care information, including code sets.

As a result of the Health Insurance Portability and Accountability Act, the AMA decided to take a detailed look at the CPT system to determine whether the then-30-year-old system was staying relevant. This analysis resulted in the establishment of the CPT-5 project. CPT was expanded very meaningfully with new nomenclature that included tracking new procedures and services. In addition, specific reporting measures that could be used in performance-based payment were built into CPT. With the expansion that resulted from the Health Insurance Portability and Accountability Act, the CMS opted to formally incorporate CPT codes in Medicare claims processing. In 2000, CPT became the national coding standard for reporting medical services and procedures. ${ }^{5}$ The AMA continues to recognize the importance of maintaining an updated and relevant CPT coding system and 
does so through resolutions in its House of Delegates, active physician involvement in systematic review of existing codes, retirement of obsolete codes, and authorship of new codes to reflect changes and innovations in medical practice. The CPT codebook remains a living document that undergoes annual updates. For example, the 2016 edition offers over 350 code changes, 140 of which are new, 134 revised, and 93 deleted. ${ }^{6}$ Therefore, CPT is a critical component in the provision of health care in 2016 and into the future.

\section{CPT COMMITTEE CONSTRUCT}

There are 2 key committees that constitute what is commonly thought of as "CPT": the CPT Editorial Panel and the CPT Advisory Committee. The CPT Editorial Panel presides over the development of new and revised codes and is important in the maintenance of code sets. ${ }^{7}$ This panel includes physicians and a variety of other stakeholders, including representatives from CMS. The CPT Editorial Panel meets 3 times per year. In contrast, the CPT Advisory Committee comprises representatives of constituent societies from the AMA House of Delegates. This is the mechanism through which the American Society of Neuroradiology (ASNR) is represented. The Advisory Committee assists the CPT Editorial Panel by proposing code set changes and, crucially, by providing insights into coding proposals submitted by other interested stakeholders (eg, other societies, insurance carriers, and industry vendors). ${ }^{7}$

\section{CPT CODE CATEGORIES}

The extensive codes in the CPT are divided into 3 categories. Category I codes constitute the CPT codes common in clinical practice. These codes require US Food and Drug Administration approval for any drugs or devices, must have demonstrated clinical efficacy supported by the peer-reviewed literature, and must be commonly practiced by multiple physicians in the United States. Category II codes are used to report quality performance initiatives and are designed to simplify participation in quality measures by diminishing administrative burdens such as medical record review. Category II codes cannot substitute for Category I codes. They are designed to facilitate data collection, tracking of performance measures, and compliance with state or federal law, but are not to be used for coding services or procedures that are ultimately billed. In keeping with the explosive increase in Physician Quality Reporting System measures, the number of category II codes is increasing. ${ }^{8}$ Category III codes were created in 2001 to track new or experimental procedures and technologies. These temporary codes are characterized with an alphanumeric descriptor (eg, 3456T). Data accumulated by these tracking codes can aid in the US Food and Drug Administration approval process. Category III codes do not need to meet the evidentiary basis that Category I codes require. Category III codes are not assigned a professional work value, and payment for these services, if any, is at the discretion of Medicare and private payers. Category III codes are by definition temporary and are only approved for a 5-year period; this can be extended once. If warranted by experimental evidence, a Category III code can be converted into a Category I code before the expiration of the initial or renewal term. If the procedure has not been proved effective by that point, the code is allowed to expire. The advisors and panel members use standard, rigorous protocols for determining medical evidence. CT perfusion imaging is an example of a current Category III code..$^{9,10}$

\section{RELATIONSHIP TO THE AMA/SPECIALTY SOCIETY RELATIVE VALUE SCALE UPDATE COMMITTEE}

Medicare has gone through multiple iterations to arrive at its current payment methodology. During the administration of President George H.W. Bush, as a result of the Omnibus Budget Reconciliation Act of 1989, CMS started to use a system of relative value units (RVUs) to calculate the cost for providing physician work, which evolved into the framework of a resource-based relative value scale. ${ }^{11}$ The AMA recruited physician input into that system. In 1992, the AMA/Specialty Society Relative Value Scale Update Committee (RUC) was convened. Codes that are established through CPT are then reviewed and debated at the RUC, which recommends suggested RVUs to CMS. Through the American College of Radiology, the radiology community has benefited from continuous representation at the RUC since this committee's inception. The ASNR gained a seat in the AMA House of Delegates in 1996 and has actively participated in the CPT and RUC meetings since then. Continued participation requires that a sufficient percentage of ASNR members maintain active AMA membership. ${ }^{12-14}$

\section{RELATIVITY ASSESSMENT WORKGROUP}

The RUC began its work in the early 1990s recommending relative values for CPT codes. Explicit in its charter was that a review of the entire resource-based relative value scale needed to be performed every 5 years. After the third such review in 2007, an ongoing review process was created at the urging of CMS. The "Five-Year Review Committee" was renamed the "Relativity Assessment Workgroup." Triggers leading to review of "potentially misvalued" codes varied; some of the screening criteria included increasing utilization of a code; change in site of service; change in the physician specialty reporting the code; or CPT codes whose valuation lacked a verifiable data trail. ${ }^{15}$

A Relativity Assessment Workgroup screen for "Codes Performed Together" has been particularly noteworthy for radiologists because many procedures had historically been reported by using a granular, building-block method known as component coding. The underlying premise of this screen is that CPT codes, which are typically reported together on a single Medicare patient on the same day of service (ie, CT abdomen and CT pelvis), may have efficiencies that should be accounted for in determining overall relative value. This screen has expanded in importance and scope over time. Whereas the original filter was set at codes reported together $95 \%$ of the time, it is now triggered when codes are performed together more than $50 \%$ of the time. ${ }^{16}$

CMS embraced the work of the Relativity Assessment Workgroup with the creation of its own screening processes for "potentially misvalued" codes; for instance, naming codes with high total expenditure. These screens frequently have targeted radiology, given the high technical component costs of advanced imaging such as PET, MR imaging, and CT. A new list of screened codes is published every July in the Medicare Physician Fee Schedule Proposed Rule as part of CMS's annual rulemaking process. Regard- 
less of why a code is "caught" by a screen or whose screen "caught" the code (Relativity Assessment Workgroup or CMS), the specialty societies are obligated to respond to the inquiry and formulate a strategy for proving appropriate valuation of both technical and professional components of procedures.

\section{WHAT HAPPENS WHEN A CPT CODE IS CAUGHT BY A RELATIVITY ASSESSMENT WORKGROUP SCREEN OR DEEMED POTENTIALLY MISVALUED BY CMS?}

Once a code has been labeled as potentially misvalued, specialty societies declaring an interest in establishing an RVU for the procedure are given the opportunity to provide evidence that the code values captured by the screen should remain untouched (ie, that they are valued appropriately or because a confounding factor would create downstream consequences if the code or code family were revised or revalued). If those arguments fail to convince the Relativity Assessment Workgroup or CMS, the next course of action is either revaluing the services via survey or revision of the CPT nomenclature or code structure (often via "bundling" of multiple discrete codes into more complex, comprehensive codes) via referral back to CPT.

CMS recognizes that these various filtering screens could lead to rank-order anomalies within groups of related codes. This has led to the loose concept of code families, which the AMA and CMS have mostly left to the specialty societies to define. The code family concept is a protective mechanism to prevent rank-order anomalies from occurring when valuing or revaluing a service. Because the various screens described above may flag some but not all procedures in a family, CMS mandates that all codes in a family that relate to the code picked up in the screen or codes that are newly created must be analyzed together. An option specialty societies can choose to take is recommending that a code or family be referred back to CPT-in other words, taking the codes off the RUC table for the time being, allowing the specialty societies to revise the code structure and definitions-before presenting them to the RUC for valuation. The ASNR actively represents neuroradiology interests (from the esoteric to the mundane) at CPT. It is noteworthy but not widely appreciated that ASNR involvement in the CPT and RUC processes is dependent upon threshold membership in the AMA.

We will explore how the changes to the CPT occur through several real-life examples below.

\section{EXAMPLES OF RECENT ASNR CODING EFFORTS Carotid Angiography: Procedure Codes Were Bundled with Radiologic Supervision and Interpretation Codes}

In 2010, numerous codes related to carotid and cerebral angiography - the procedural codes describing catheterization and injection and the codes describing radiologic supervision and interpretation codes-were identified via the Codes Performed Together Screen. Per above, the entire family of codes needed to be revised to prevent possible rank-order anomalies. The new code bundle needed to include both the procedural and interpretive components. The CPT Editorial Panel, with input from multiple constituent societies, including ASNR, approved a new series of codes for these services in 2012. These new codes, which bundled the procedure codes with the supervision and interpre- tation codes, then were sent to the RUC for valuation recommendations. ${ }^{17}$

This code-bundling process resulted in a significant drop in RVUs and, therefore, physician reimbursement, beginning in January 2013. For example: a single vessel-selective diagnostic angiogram of the right internal carotid artery would have a CMS value of 7.60 RVUs in 2012 with standard component coding (including typical reductions for second and subsequent procedures); the single bundled code for that procedure and interpretation has been valued at 6.50 RVUs since 2013, a reduction of $15 \%$. A standard 4-vessel angiogram previously valued at 18.22 RVUs is now 14.25 RVUs, a reduction of $22 \% .{ }^{17}$

The negative impact of code bundling on diagnostic radiology was probably most severely felt when bundled codes for CT of abdomen and CT of pelvis were mandated by CMS, also based on the Codes Performed Together Screen. Using the intravenous contrast-enhanced procedures for this example (74160 and 72193), the 2 codes separately reported in 2011 and before were valued at 2.42 RVUs; the combined code 74177 is valued at 1.82 RVUs, a reduction of $25 \%$.

Although beyond the scope of this article, practice expense reimbursement is also affected by code bundling, mainly because of reductions in room times, technologist labor, and nurse labor.

\section{Myelography: Procedure Code Was Bundled with Radiologic Supervision and Interpretation Codes}

Another classic procedure performed by neuroradiologists was caught in the Codes Performed Together Screen because of the near-universal association between injection and supervision and interpretation codes. ASNR and the American College of Radiology revised the code set and presented the new bundled codes (as well as the original stand-alone codes) to the RUC for valuation. CPT 2015 introduced 4 new bundled codes for myelography in the setting of the same physician performing the injection, supervising the procedure, and interpreting the images. If separate providers perform the procedure and do the interpretation, then the nonbundled original codes are used; hence, the existing codes were maintained (ironically adding more codes to the myelography code set).

It is important to emphasize that the CPT descriptor for myelography includes a thorough radiographic (plain film) examination of the spine after intrathecal injection of iodinated contrast media, involving assessment of static structures, such as the spinal canal and each exit foramen, and real-time assessment of contrast injection and its flow dynamics under direct visualization. ${ }^{18}$ It is not appropriate to code for a formal myelogram when $\mathrm{x}$-ray imaging is simply used to document the intrathecal location of needle placement and contrast injection.

Coincidentally, in the same year that ASNR was revising the myelography code set and presenting it to the RUC, the National Correct Coding Committee proposed adding an edit that would deny billing of postmyelography CT on the same day as a radiographic myelogram, independent of the number of providers. CMS raised concerns that myelography and CT with intrathecal contrast media were duplicative procedures. Stakeholders led by the neuroradiology subspecialty societies provided clarification to CMS, which was accepted, and the edit was not pursued further 
Examples of comparative code terminology from various coding sources ${ }^{20,21}$

\begin{tabular}{lll}
\hline Source & Vertebroplasty (Injection Only) & Vertebral Augmentation (Injection and Mechanical Device) \\
\hline ACR & Vertebroplasty, acrylic vertebroplasty & Balloon kyphoplasty, balloon-assisted vertebroplasty \\
AMA CPT & Percutaneous vertebroplasty & Percutaneous vertebroplasty augmentation including cavity \\
& & creation using mechanical devices, kyphoplasty \\
FDA & Vertebroplasty & Kyphoplasty \\
CMS & Vertebroplasty & Kyphoplasty \\
\hline
\end{tabular}

Note:-ACR indicates American College of Radiology; FDA, US Food and Drug Administration.

beyond adding the -59 modifier when CT of the spine with contrast media is used with the myelography codes. ${ }^{18}$

\section{Vertebral Augmentation: Procedure Code Was Bundled with Radiologic Supervision and Interpretation Codes}

Similar to myelography, the effects of bundling extended to the vertebral augmentation code family. The CPT/RUC Joint Workgroup recommended that the injection and interpretation codes for vertebroplasty and vertebral augmentation or sacral augmentation (sacroplasty) be bundled. In February 2014, the CPT Editorial Panel replaced the 8 Category I codes with 6 new comprehensive codes to bundle injection and imaging guidance/ interpretation: 22510-22512 for vertebroplasty (cervicothoracic, lumbosacral, and each additional level) and 22513-22515 for kyphoplasty (thoracic, lumbar, and each additional level).

The sacral procedures that were previously coded as Category III codes (0200-01T) were revised to be included under lumbosacral vertebroplasty. Similarly, cervical vertebroplasty was revised to be coded as cervicothoracic. The language used in these procedures can be confusing for coders (Table), and we provide in the reference list a CPT Assistant article with language to help clarify the differences and provide historical context. ${ }^{19}$

\section{Scoliosis Plain Film Series: Code Revision and Code Bundling}

The family of scoliosis plain film codes was identified as inconsistent with other plain film codes; some were descriptive of the views, whereas others were defined by the number of views. To reduce confusion, the codes were revised in October 2014 so that the scoliosis code family is now entirely defined by the number of views obtained: 1 view; 2 or 3 views; 4 or 5 views; and 6 or more views (72801, -82, -83, and -84, respectively). This standardized hierarchy and nomenclature is now in step with most other radiography code descriptors. ${ }^{20}$

\section{Fetal MR Imaging: Creation of a New Code}

Historically, imaging of the fetus was reported by using an unlisted code or the pelvic MR imaging codes 72195-72197. After an e-mail from a member of the Society of Pediatric Radiology to the AMA, advisors from the ASNR, American College of Radiology, American Roentgen Ray Society, and Society of Pediatric Radiology jointly crafted a new coding proposal for fetal MR imaging to the CPT Editorial Panel in February 2015. The new fetal MR codes_-"Magnetic resonance (eg, proton) imaging, fetal, including placental and maternal pelvic imaging when performed; single or first gestation" (74712) and an add-on code: "Magnetic resonance (eg, proton) imaging, fetal, including placental and maternal pelvic imaging when performed; each additional gestation (List separately in addition to code for primary procedure)" (74713)—were accepted into the CPT canon.
Having a discrete CPT code for fetal MR imaging allows members of specialty societies to articulate the differences in physician work and technical expense relative to standard pelvic MR imaging. While presenting to the RUC in April 2015, the societies' advisors explained that unlike routine pelvic MR imaging, fetal MR imaging is complicated by continuous patient movement, complex anatomy, and intrinsic multiorgan evaluation. ${ }^{20}$

\section{DOWNSTREAM CONSEQUENCES OF NEW OR REVISED CPT CODES}

Radiologic societies are cognizant of the downstream effect code revisions and code bundling has on the radiology community. Code bundling not only leads to revisions in Medicare payments, but also requires renegotiation of private insurance contracts. Occasionally, the new bundled service, which by default lacks the granularity of the individual parent codes, may not fully capture how a service is performed in the community. The less granular bundled code can lead to unintended confusion and inequities in the reported cost to perform the procedure.

A recent example of this confusion and lack of granularity is represented by a multiyear project to bundle conscious sedation and anesthesia services into a base procedure (ie, interventional radiology procedure, colonoscopy, etc), where the same physician performing the base procedure typically performs sedation. CMS and the RUC define "typical" as occurring greater than $50 \%$ of the time. If a service such as conscious sedation is typical, then it should not be billed separately and should be bundled into the base code being billed by the physician performing the procedure. Unfortunately, the loss of granularity makes it very difficult to bill anesthesia services or conscious sedation in atypical situations or when an anesthesiologist is required. It also leads to inequities in what was paid for when providing conscious sedation or anesthesia services, depending on what the base procedure code was and not on the actual service. This confusion has led to a new multiyear project of unbundling conscious sedation and anesthesia services from all previous base codes. This project provides an example of the unintended consequences and potential harms of bundling.

Developing or revising CPT codes not only requires substantial effort to accurately and appropriately define and categorize medical procedures, but also involves significant strategic considerations, diplomacy, and collaboration. Because code development at the CPT Editorial Panel leads directly to discussions of valuation at the RUC, it is important that the structure of new and revised medical procedure codes appropriately captures the imaging work performed by radiologists and neuroradiologists as part of those procedures. Different specialty societies may view the work of imaging differently in areas where overlap could exist, such as intraoperative MR imaging, functional MR imaging, and 
carotid stent placement, or in non-neurologic codes such as CT colonography or coronary CTA. Accurately describing procedures in a way that fully captures the work of image guidance or interpretation and that allows for rapid technologic progress is a team effort and requires an open-minded, collaborative approach.

\section{CONCLUSIONS}

CPT began 50 years ago as an effort to accurately report what was largely a group of surgical services and bill for them more precisely. The CPT system has grown in scope over the years and has seen variable granularity, reflecting the innovations of new procedures, changes in legacy technology, and retirement of obsolete approaches. More recent pressures have come in the form of bundling and code revisions by CMS and the AMA's RUC. An understanding of the CPT coding history and current direction is important as providers navigate these new waters of health care reimbursement.

Disclosures: Jacqueline Bello-OTHER RELATIONSHIPS: serves as the ASNR's Assistant Specialty Advisor to the CPT Panel (as described in the article). Joshua HirschUNRELATED: Consultancy: Medtronic, Comments: interventional spine, Codman Neurovascular, Comments: Data Safety and Monitoring Board work.

\section{REFERENCES}

1. Thorwarth WT Jr. CPT: an open system that describes all that you do. J Am Coll Radiol 2008;5:555-60 CrossRef Medline

2. Thorwarth WT Jr. From concept to CPT code to compensation: how the payment system works. J Am Coll Radiol 2004;1:48-53 CrossRef Medline

3. Hirsch JA, Leslie-Mazwi TM, Nicola GN, et al. Current procedural terminology; a primer. J Neurointerv Surg 2015;7:309-12 CrossRef Medline

4. HIPAA for Professionals. Services UDoHaH. http://www.hhs.gov/ hipaa/for-professionals/index.html. Accessed March 17, 2016

5. CPT Process - How a Code Becomes a Code. American Medical Association. http://www.ama-assn.org/ama/pub/physician-resources/ solutions-managing-your-practice/coding-billing-insurance/cpt/ cpt-process-faq/code-becomes-cpt.page. Accessed March 15, 2015

6. 2016 AMA CPT Professional Edition Book. American Medical Association. https://www.aapc.com/medical-coding-books/2016/2016cpt-book.aspx?gclid=CO2y_cXUy8sCFYY2gQodPYwIog. Accessed March 17, 2016

7. CPT - Current Procedural Terminology. American Medical Association. http://www.ama-assn.org/ama/pub/physician-resources/ solutions-managing-your-practice/coding-billing-insurance/cpt/ cpt-editorial-panel.page. Accessed March 15, 2016

8. CPT - Category II Codes. American Medical Association. http:// www.ama-assn.org/ama/pub/physician-resources/solutions-managingyour-practice/coding-billing-insurance/cpt/about-cpt/category-iicodes.page. Accessed March 15, 2016

9. Hirsch JA, Schaefer PW, Romero JM, et al. Comparative effectiveness research. AJNR Am J Neuroradiol 2014;35:1677-80 CrossRef Medline

10. CPT - Category III Codes. American Medical Association. http:// www.ama-assn.org/ama/pub/physician-resources/solutions-managingyour-practice/coding-billing-insurance/cpt/about-cpt/category-iiicodes.page. Accessed March 15, 2016

11. Manchikanti L, Singh V, Caraway DL, et al. Medicare physician payment systems: impact of 2011 schedule on interventional pain management. Pain Physician 2011;14:E5-E33 Medline

12. Hirsch JA, Silva E, 3rd, Nicola GN, et al. The RUC: a primer for neurointerventionalists. J Neurointerv Surg 2014;6:61-64 CrossRef Medline

13. The RVS Update Committee. American Medical Association. http:// www.ama-assn.org/ama/pub/physician-resources/solutions-managingyour-practice/coding-billing-insurance/medicare/the-resourcebased-relative-value-scale/the-rvs-update-committee.page. Accessed March 15, 2016

14. Donovan WD. The resource-based relative value scale and neuroradiology: ASNR's history at the RUC. Neuroimaging Clin N Am 2012;22:421-36 CrossRef Medline

15. Silva $\mathrm{E} 3 \mathrm{rd}$. New codes from a new source: the rolling five-year review. J Am Coll Radiol 2010;7:10-12 CrossRef Medline

16. Hirsch JA, Donovan WD, Leslie-Mazwi TM, et al. Component coding and the neurointerventionalist: a tale with an end. J Neurointerv Surg 2013;5:615-19 CrossRef Medline

17. Donovan WD, Leslie-Mazwi TM, Silva E 3rd, et al. Diagnostic carotid and cerebral angiography: a historical summary of the evolving changes in coding and reimbursement in a complex procedure family. J Neurointerv Surg 2014;6:712-17 CrossRef Medline

18. Chokshi FH, Tu RK, Nicola GN, et al. Myelography CPT coding updates: effects of 4 new codes and unintended consequences. AJNR Am J Neuroradiol 2016;37:997-99 CrossRef Medline

19. License for Use of Physicians' Current Procedural Terminology, Fourth Edition (CPT). Services CfMaM. http://www.cms.gov/ medicare-coverage-database/details/lcd-details.aspx. Accessed March 15,2016

20. American Medical Association. 2016 AMA CPT ${ }^{\circledR}$ Professional Edition Book. Chicago: American Medical Association; 2016

21. American Medical Association. Coding Update: Percutaneous Vertebroplasty and Vertebral Augmentation. Vol 1. Chicago: American Medical Association; 2015 